Copyright 2009 Society of Photo-Optical Instrumentation Engineers.

This paper was published in Proceedings of SPIE, vol. 7260, Medical Imaging 2009: Computer Aided Diagnosis and is made available as an electronic reprint with permission of SPIE. One print or electronic copy may be made for personal use only. Systematic or multiple reproduction, distribution to multiple locations via electronic or other means, duplication of any material in this paper for a fee or for commercial purposes, or modification of the content of the paper are prohibited. 


\title{
Bronchial Segment Matching in Low-dose Lung CT Scan Pairs
}

\author{
Jaesung Lee ${ }^{a}$, Anthony P. Reeves ${ }^{a}$, \\ David F. Yankelevitz ${ }^{b}$, and Claudia I. Henschke ${ }^{b}$ \\ ${ }^{a}$ School of Electrical and Computer Engineering, Cornell University, Ithaca, NY; \\ ${ }^{b}$ Department of Radiology, Weill Cornell Medical College, New York, NY
}

\begin{abstract}
Documenting any change in airway dimensions over time may be relevant for monitoring the progression of pulmonary diseases. In order to correctly measure the change in segmental dimensions of airways, it is necessary to locate the identical airway segments across two scans. In this paper, we present an automated method to match individual bronchial segments from a pair of low-dose CT scans. Our method uses the intensity information in addition to the graph structure as evidences for matching the individual segments. 3D image correlation matching technique is employed to match the region of interest around the branch points in two scans and therefore locate the matching bronchial segments. The matching process was designed to address the differences in airway tree structures from two scans due to the variation in tree segmentations. The algorithm was evaluated using 114 pairs of low-dose CT scans (120 kV, $40 \mathrm{mAs})$. The total number of segments matched was 3591, of which 99.7\% were correctly matched. When the matching was limited to the bronchial segments of the fourth generation or less, the algorithm correctly identified all of 1553 matched segments.
\end{abstract}

Keywords: Lung, CT, airway, bronchial segment

\section{INTRODUCTION}

The airway dimensions are affected by pulmonary diseases such as COPD. Documenting any change in airway dimensions over time may be relevant for monitoring the progression of such diseases. Further, it may be necessary to assess the effect on airway dimensions of therapeutic responses. The change in airway dimensions, such as lumen diameter and wall thickness, may be documented over individual airway segments across timeseparated CT scans. $[1,2]$ In order to correctly measure the change in segmental dimensions, it is necessary to locate the identical segments across two scans of a same patient.

Individual segments of a human airway have tubular structure, and the centerline of this tube may be curved to a certain extent. Each airway segment is characterized by low-intensity lumen area and high-intensity airway wall that surrounds the lumen. Automated matching of bronchial segments in low-dose CT scans is a challenging task due to the complicated nature of airway tree structure and the amount of noise present in the image. Segment matching is especially challenging for smaller airways because the airway tree becomes more naive for higher-generation segments.

An earlier approach to this problem presented by Tschirren et al. [3] used the information from the skeletal apperance of the airway tree, such as inheritance relationship, length, and orientation angle, for matching the segment pair.[3] Their method used the segmental information to build the association graph, with a vertex representing a segmental match candidates across two scans and an edge between two vertices representing the identical relationship between two candidates within the same tree for the connected vertices. The matching segments were then determined by finding the maximum clique from the established association graph.

In this paper, an automated airway segmental matching method is presented which uses the intensity information around the branch points by using 3D image registration technique in addition to the skeletal graph

Further author information: (Send correspondence to Jaesung Lee) Jaesung Lee: E-mail: j1569@cornell.edu, Telephone: 16072550963

Medical Imaging 2009: Computer-Aided Diagnosis, edited by Nico Karssemeijer, Maryellen L. Giger Proc. of SPIE Vol. 7260, 72600A · C 2009 SPIE · CCC code: 1605-7422/09/\$18 · doi: 10.1117/12.812024 

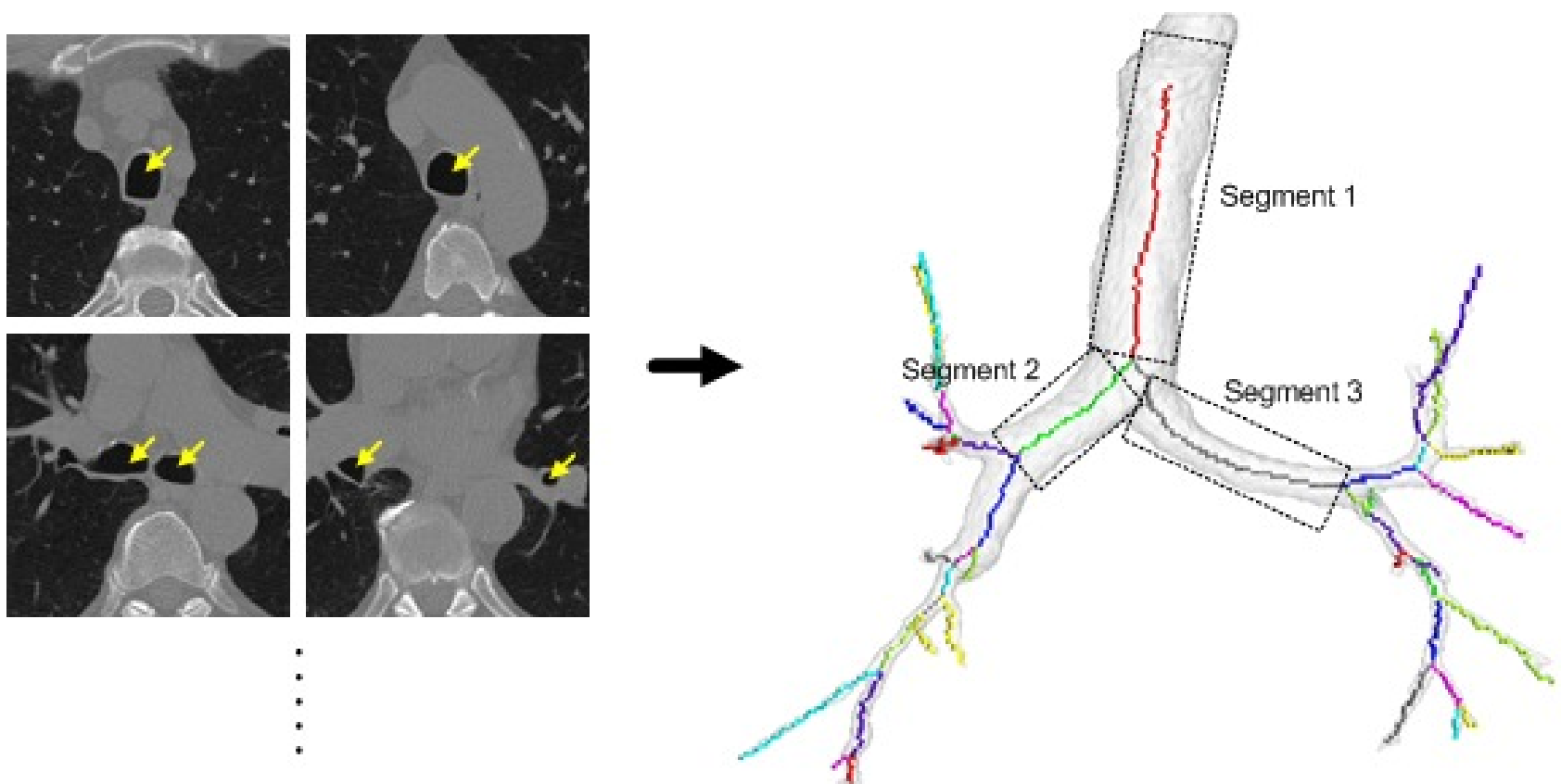

Figure 1. Identification of individual segments in an airway tree. On the left CT slices are shown with the arrows indicating major airways. On the right the identified airway segments from a single CT scan is shown. The boxes are placed on the trachea and two main bronchi, and the centerlines of the segments are colored with different colors.

structure. Using the intensity information in addition to the tree's skeletal structure should result in more robust matching of airway segments, as it depends less on the predetermined properties of airway tree drawn from training data.

Image registration is widely employed in various modalities of medical images such as X-ray, computed tomography (CT), magnetic resonance (MR), and ultrasound. $[4,5,6,7]$ The need for image registration arises in clinical settings as integration of data obtained from the separate images is often desired. [8] The proposed segment matching algorithm performs 3D image registration on the ROIs around the end points of individual airway segments in order to assess the similarity of two regions.

\section{METHOD}

\subsection{Overview}

An input to the matching algorithm is the skeleton of the segmented airway tree. The skeletal representation of the airway tree is obtained using a method outlined in [1]. The airway lumen is segmented from the CT images using adaptive 3D region growing, and 3D thinning algorithm is used to get the skeleton of the airway tree. Our matching algorithm consists of two stages; first, individual airway segments are located separately in two scans, then the corresponding segments are matched across identified segments.

\subsubsection{Segment Identification}

First, individual segment needs to be identifed for each airway tree, as shown in Figure 1. Starting with the trachea, the centerline of the airway tree is traversed to locate the start and end points of individual airway segment. When a bifurcation of the centerline is encountered, the algorithm ends the current segment and starts new segments. A tree data structure is used to represent the entire airway structure. Each node in the tree corresponds to an airway segment, and the subsequent segments are represented as children nodes. 


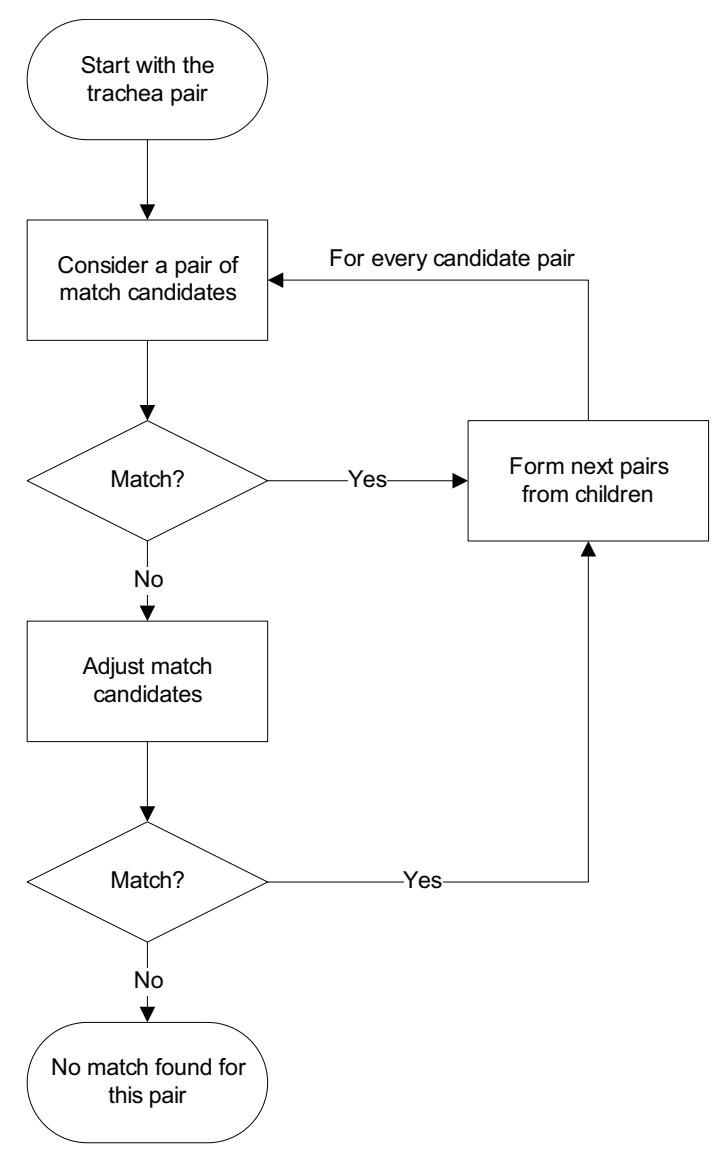

Figure 2. Flowchart showing the matching process for the entire tree once the segments are identified separately in each scan. The matching starts with the trachea pair from the two scans and proceeds down the tree.

\subsubsection{Segment Matching}

For each airway segment, the start point is the end point of the parent segment, and therefore only an end point needs to be matched. The entire matching process is outlined as a flowchart in Figure 2.

The branch points are matched starting from the end point of the trachea. Two candidate branch points, once identified as match candidates, need to be evaluated for correct matching. The matching criteria is discussed in Sections 2.2 and 2.3. Once a pair of match candidates is matched, the next pair of candidates need to be determined. This process is described in Section 2.4. When the candidates fail to match on the first try, then the matching is attempted once more with adjusted match candidates. This process is described in Section 2.5.

\subsection{ROI Matching}

To determine if two match candidates have identical end points, a 3D image matching algorithm was used. First, a spherical region of interest is established around the end point of each segment. The size of ROI (diameter of sphere) is determined based on the branch generation for the given segments and is chosen to include the entire airway and some lung parenchyma region around this airway. Specifically, the diameters were set to $35 \mathrm{~mm}$ for the first two generations, $20 \mathrm{~mm}$ for the next two generations, and $15 \mathrm{~mm}$ for the remainder of the segments.

A 3D image correlation matching algorithm is then used to evaluate the similarity of the two end points (match candidates). The MSE is computed between two spherical region as shown in Equation 1, where the summation terms only apply to the spherical region.

$$
M S E(c 1, c 2)=\sum_{k} \sum_{j} \sum_{i}(c 1(i, j, k)-c 2(i, j, k))^{2},
$$




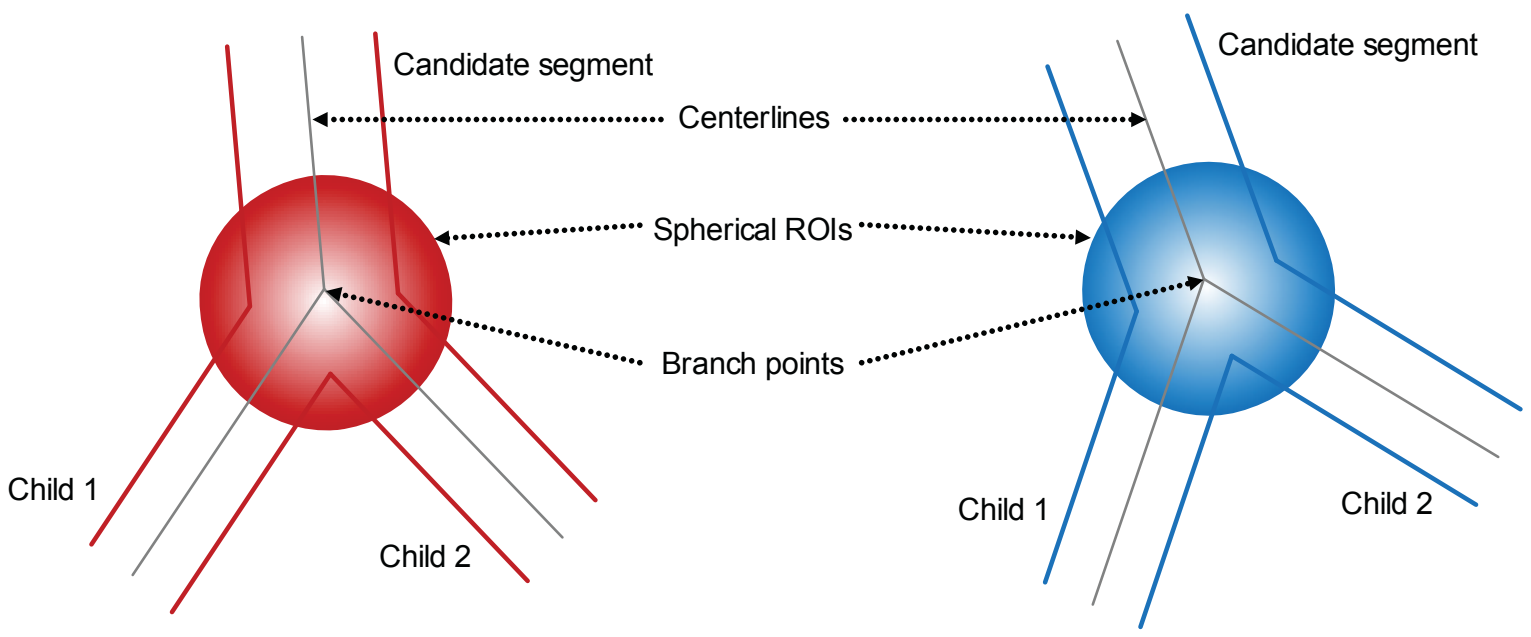

Figure 3. Matching spherical ROIs. The diagram shows spherical ROIs placed on the end points of the match candidate segments from tree 1 and tree 2 . The given ROIs are registered to one another to minimize MSE between them.

where $c 1$ and $c 2$ are the spherical ROIs around each candidate's end point.

The optimal translation and rotation values were found to minimize the MSE between the two ROIs. (i.e. two ROIs were registered to one another) The translation in each of $\mathrm{x}, \mathrm{y}$, and $\mathrm{z}$ directions was limited to $3.5 \mathrm{~mm}$, and the rotation around each of three axis was limited to 10 degrees.

Since two trees are from the same patient and have small variation in their orientations, the assumption was that the minimum MSE would be reached at small translation and rotation values. In other words, it was assumed the best match location is very close to the starting location (zero translation and zero rotation) if the candidiates were the identical end points in the first place. Therefore, hill climbing approach was used to search for the minimum MSE, where the search proceeds toward the steepest decrease in MSE until a local minima is found.

\subsection{Matching Criteria}

The registration of two candidate ROIs yields a minimum MSE. The candidates were classified as a match or non-match based on this MSE value, as shown in Equation 2.

$$
\text { Matching decision }=\left\{\begin{array}{l}
\text { Match if } M M S E<=\text { Threshold(gen })+ \text { tolerance, } \\
\text { Non-match if } M M S E>\text { Threshold(gen })+ \text { tolerance, }
\end{array}\right.
$$

where $M M S E$ is the minimum MSE value resulting from the registration of two ROIs, and $g e n$ is the generation of a given airway segment.

The threshold was determined based on the registration of the trachea's end points, as the trachea can be matched robustly in majority of cases. Setting a threshold this way prevents overfitting of the preset parameter (MSE threshold) to the training data. When making a matching decision, some level of tolerance was added to the threshold in order to compensate for MSE variations in different pairs of ROIs.

Different thresholds were used for the segments in different generations since the diameters of the ROIs were varied based on the branch generation. The spherical ROIs with different sizes (35 mm, $20 \mathrm{~mm}$, and $15 \mathrm{~mm})$ were established around the end point of the trachea, and the minimum MSE values were obtained and used as the threshold for each ROI size.

The algorithm's parameters, including the ROI size, level of tolerance, and maximum allowed translation and rotation, have been fine-tuned using 6 cases that have been set aside for training. 


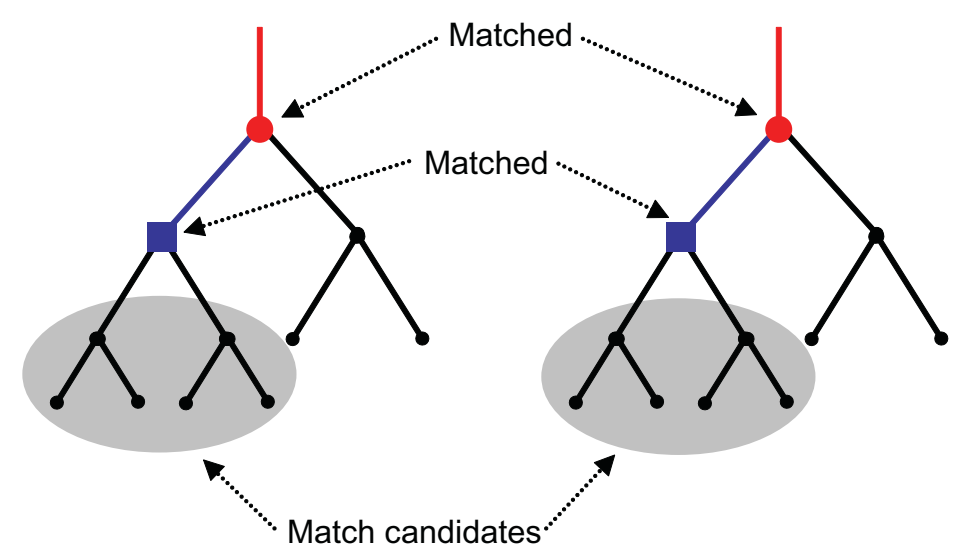

Figure 4. Tree diagram showing how the candidate pair is formed in two airway trees. Each node in the tree represents an airway segment. Once a segment pair is matched (rectangular nodes), new match candidate pair is searched from a pool of match candidates (gray area).

\subsection{Candidate Determination}

Once a pair of end points is matched, the search space for the children segments are constrained to the subtree following the matched node. The next pair of candidates are chosen from the children of the end points that were just matched (Figure 4). In the candidate selection process, it is necessary to determine which children in tree 1 and tree 2 should be paired as a match candidate.

For example, consider a case where the number of children is two, which is in fact the most frequent case. Given the matched segments $\mathrm{m} 1$ and $\mathrm{m} 2$, there would be the children nodes $\mathrm{c} 1 \mathrm{a}$ and $\mathrm{c} 1 \mathrm{~b}$ of the parent $\mathrm{m} 1$ and the children nodes $\mathrm{c} 2 \mathrm{a}$ and $\mathrm{c} 2 \mathrm{~b}$ of the parent $\mathrm{m} 2$. In this case, there are two possible scenarios of match candidate pairs. The first scenario would be c1a-c2a and c1b-c2b pairs, and the second would be c1a-c2b and c1b-c2a pairs.

The goal is to pair the candidates that are most likely to be the same segments. To do so the orientation angle of each airway segment is taken into account. The angle between the segments in each of possible pairs is computed for all possible scenarios. The scenario with the minimum angle difference is chosen by the algorithm, and the matching proceeds with this scenario for comparing the branch points of the children segments. This decision process is represented in Equation 3.

$$
\text { Decision }=\operatorname{argmin}_{X \in S}\{\min \{\operatorname{Angle}(p), p \in X\}\},
$$

where $X$ is a scenario in a set $S$ of all possible scenarios, $p$ is a pair of match candidates (from tree 1 and tree $2)$, and $\operatorname{Angle}(p)$ is the angle between a candidate pair $p$.

\subsection{Non-match (Extended Search)}

This section describes how the algorithm proceeds when a chosen candidate is determined to be a non-match. A non-match means that the candidate segments have the correct starting point locations, since these have been matched as the end points of their parents, but have different end points.

Assuming that a given candidate pair was correctly chosen in candidate determination step, the only reason for a non-match would be the difference in segment length. The variation in airway tree segmentation for two scans can cause such a case. Due to incomplete segmentation, a candidate segment from tree 1 could be missing a sub-branch that would otherwise originate from the middle of the segment, while another candidate segment from tree 2 is terminated by this sub-branch. In other words, there can be a branch point that is identified 


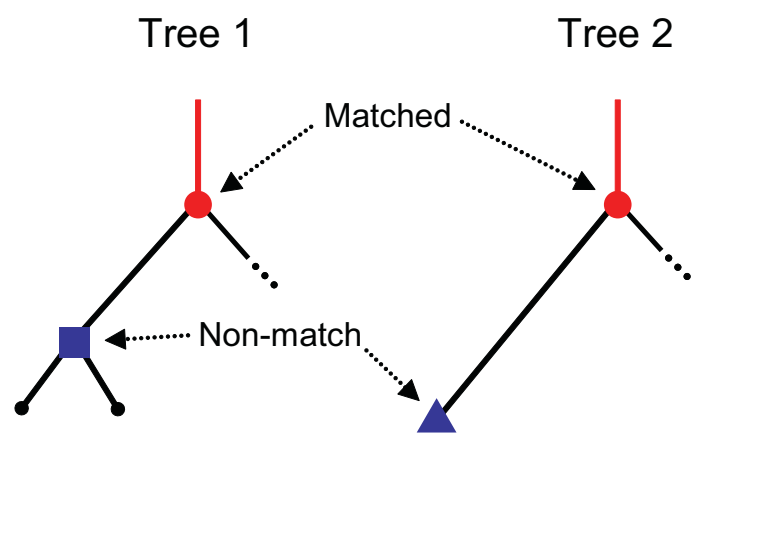

(a)
Tree 1

Tree 2

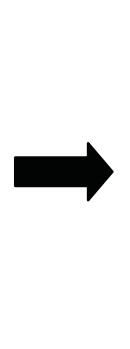

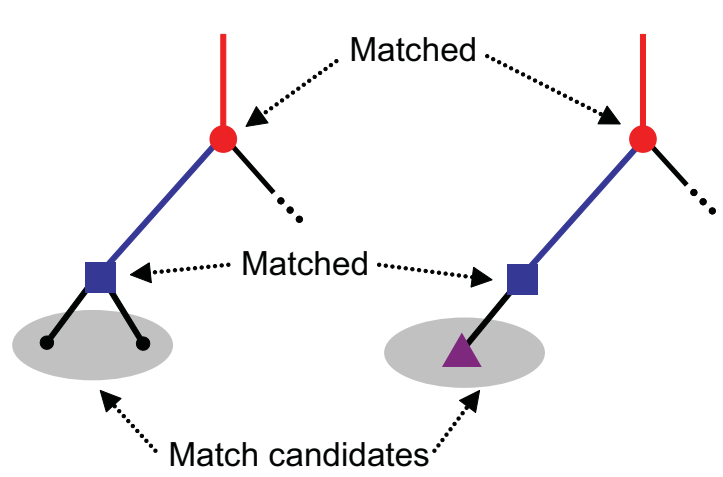

(b)

Figure 5. Insertion of dummy branch point. Tree diagram shows the two airway tree structures where each node represents an airway segment. When the algorithm fails to match a pair of match candidates as shown in (a), a dummy branch point is introduced on a longer segment (segment in tree 2 in this case). The newly introduced segment is then matched, and the matching process continues.

by the automated segmentation in one scan and not recognized in another scan. This situation is illustrated in Figure 5a.

In such a case, our algorithm introduces a dummy branch point for the tree that is missing a branch point. The algorithm creates new pair of candidate segments by cutting the longer segment to the length of the shorter segment. Once new candidates are matched, the matching process continues by determining next candidate pairs from the children of the matched segments. Since there would be only one child of the cut segment (the remainder of the segment), only one pair of candidate segments is formed for this case. Figure 5b shows this process.

\section{EXPERIMENTS}

\subsection{Experimental Design}

An experiment was designed to evaluate the accuracy of the segment matching between time-separated CT scans of a same patient. Low-dose CT scans of patients who have developed no symptoms in the interim were matched using the automated method. The outcome of the matching was visually verified using 3D interactive viewer (Figure 6).

The accuracy of the algorithm was quantified by counting the number of correctly matched segments and dividing it by the total number of matched segments. The accuracy of the automated matching was computed for the identified segments of all generations as well as for the first four generations.

\subsection{Dataset}

The dataset used for the experiments included 120 whole-lung CT scan pairs. All scans had the slice thickness of $1.25 \mathrm{~mm}$ and were taken with low radiation dose $(120 \mathrm{kV}, 40 \mathrm{mAs})$. Six cases were used for fine-tuning algorithm parameters, and the remaining 114 scan pairs were used to evaluate the accuracy of the matching algorithm.

\section{RESULTS}

Table 1 shows the average number of segments that were identified in two scans and the average number of segments matched by the algorithm. Approximately $67 \%$ of the segments identified separately in each scan were matched by the algorithm. 


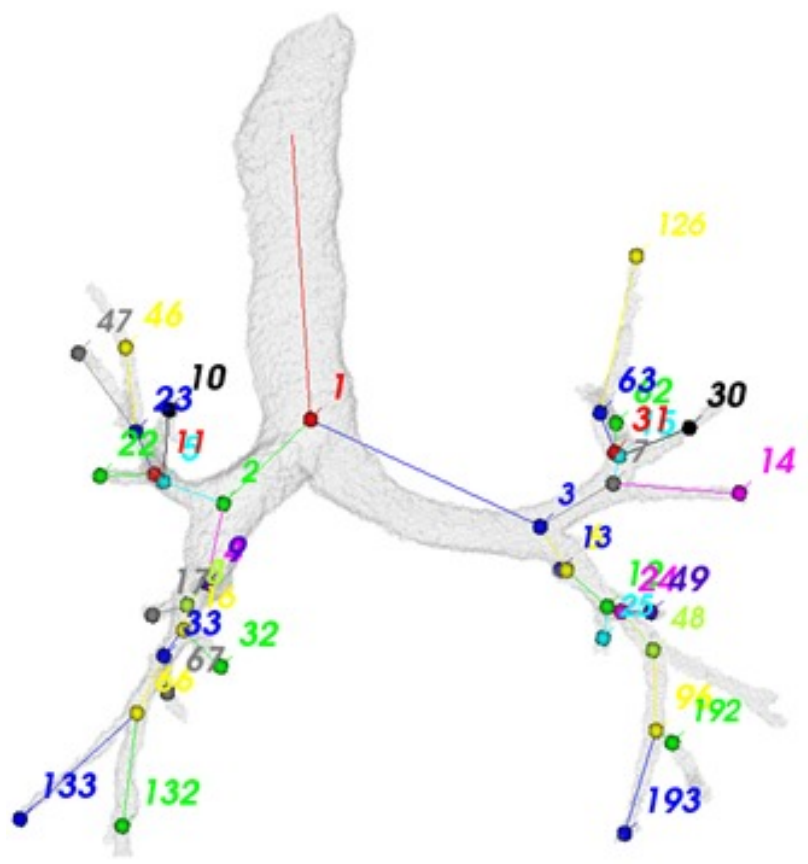

(a)

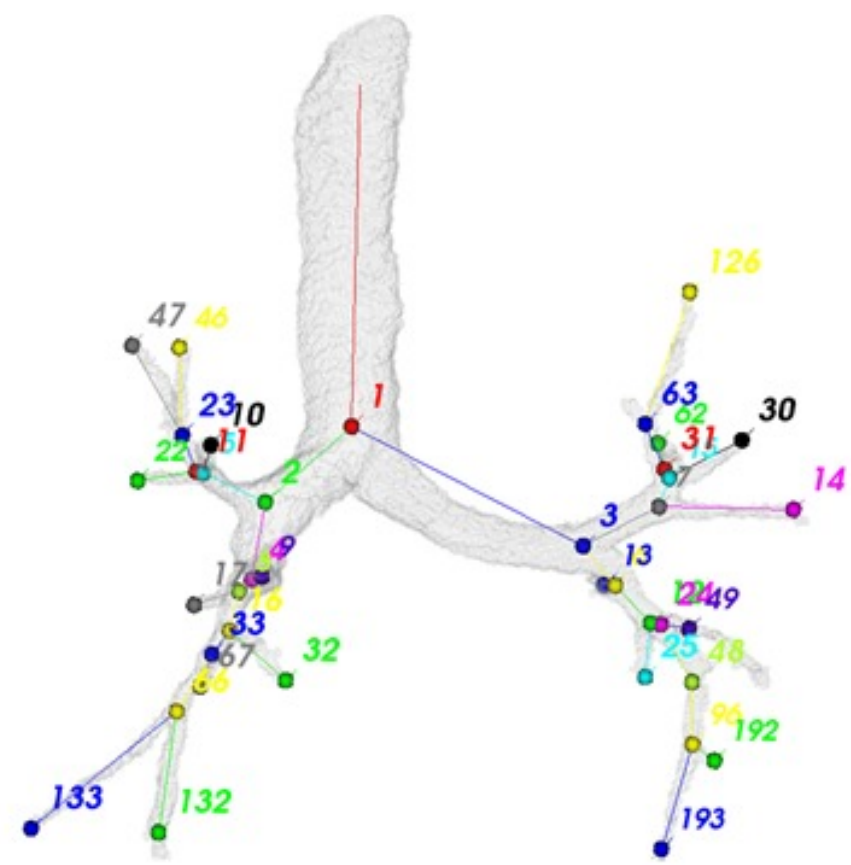

(b)

Figure 6. 3D visualization of matched segments in an entire airway tree. The segments matched across two trees are shown for both tree 1 (a) and tree 2 (b). Straight lines connecting start and end points are drawn, and the spheres are placed on the end points of the segments. The same colors are used in both trees for the matched segment. Each segment is labeled with an unique number at its end point.

Table 1. Average Number of Segments per Case

\begin{tabular}{lll}
\hline Identified in tree 1 & Identified in tree 2 & Matched \\
\hline \hline 46 & 47 & 31 \\
\hline
\end{tabular}

Table 2 shows the result of running the automated matching on 114 scan pairs. A total of 3591 bronchial segments were matched across the scans, which corresponds to 31 segments on average per patient. The accuracy of matching was $99.7 \%$, as 3581 segments were correctly matched. When the matching was limited to the bronchial segments of fourth generation or less, the matching accuracy increased to $100 \%$.

Table 2. Accuracy of Automated Segment Matching

\begin{tabular}{llll}
\hline Generations & Matched segments & Correct match & Avg. match per case \\
\hline \hline All & 3591 & $3581(99.7 \%)$ & 31 \\
\hline up to 4th & 1553 & $1553(100 \%)$ & 13 \\
\hline
\end{tabular}

\section{DISCUSSION}

The proposed algorithm uses 3D image correlation technique to register the ROIs around the end points of airway segment pair. The method uses the image registration technique for the purpose of assessing the similarity of two ROIs rather than for mapping one ROI to another. Using intensity information present in CT images makes the algorithm more robust than using the skeletal structure of the airway tree alone. 
The main cause of false matches was similar image appearances within ROIs of match candidates. It is possible to get low MSE for non-identical segments when the image information is similar around the end points, especially for the small branches toward the higher generation. However, as the results show, the algorithm was able to keep the false positive rate very small $(0.3 \%)$.

The algorithm only matched 31 segments on average per case although more than 40 segments have been identified in individual trees. One reason is that in many cases a segment identified in first tree is not identified in second tree due to variation in airway segmentation from two CT scans. Another reason is that once the algorithm fails to find a match for a given segment, all descendant segments in the subtree originating from that segment cannot be matched.

Future work will focus on consistant segmentation of the airway tree structure across different scans of a same patient, as well as new match candidate selection mechanism that can be used when no match is found for a particular segment.

\section{CONCLUSION}

Accurate matching of airway segments across a scan pair is an essential step for assessing change in airway's segmental dimensions. In this paper, the automated algorithm to identify and match bronchial segments in a pair of low-dose CT scans is presented.

The intensity information around the end point was used as an evidence for matching segments by employing a correlation-based 3D image registration. The use of intensity information makes the algorithm robust to the variation in skeletal appearances of airway tree pair. The method has been evaluated with 3591 segments from 114 repeat CT scan pairs, and the results indicate that the algorithm performs with high accuracy.

\section{ACKNOWLEDGMENTS}

This research was supported in part by NIH grant R33CA101110 and the Flight Attendants' Medical Research Institute. Drs. Henschke, Yankelevitz and Reeves are co-inventors on a patent and other pending patents which are owned by Cornell Research Foundation (CRF) who non-exclusively licenses them to General Electric for technology involving detection and characterization of nodules in many medical situations, including measurement of nodules. They receive royalties from CRF pursuant to Cornell policy, which in turn is consistent with the Bayh-Dole Act. They are also inventors on a pending patent application for lesion measurement. Dr. Yankelevitz is an inventor on a pending patent owned by PneumRx related to biopsy needles, serves as a medical advisor to them, and holds an equity interest in PneumRx.

\section{REFERENCES}

[1] Lee, J., Reeves, A., Fotin, S., Apanasovich, T., and Yankelevitz, D., "Human airway measurement from CT images," Proceedings of SPIE Medical Imaging 6915, 691518 (2008).

[2] Lee, J., Reeves, A. P., Yankelevitz, D. F., and Henschke, C. I., "Skewness reduction approach for measuring airway wall thickness," International Journal of Computer Assisted Radiology and Surgery 3, S50-S52 (2008).

[3] Tschirren, J., McLennan, G., Palagyi, K., Hoffman, E., and Sonka, M., "Matching and anatomical labeling of human airway tree," IEEE Transactions on Medical Imaging 24(12), 1540-1547 (2005).

[4] Cox, R. and Jesmanowicz, A., "Real-Time 3D Image Registration for Functional MRI," Magnetic Resonance in Medicine 42, 1014-1018 (1999).

[5] Davatzikos, C., Prince, J., and Bryan, R., "Image registration based on boundary mapping," IEEE Transactions on Medical Imaging 15(1), 112-115 (1996).

[6] Krucker, J., LeCarpentier, G., Fowlkes, J., and Carson, P., "Rapid elastic image registration for 3-D ultrasound," IEEE Transactions on Medical Imaging 21(11), 1384-1394 (2002).

[7] Maes, F., Collignon, A., Vandermeulen, D., Marchal, G., and Suetens, P., "Multimodality image registration by maximization of mutualinformation," IEEE Transactions on Medical Imaging 16(2), 187-198 (1997).

[8] Maintz, J. and Viergever, M., "A survey of medical image registration," Medical Image Analysis 2(1), 1-36 (1998). 